\title{
SOFIA observations of CO (12-11) emission along the L1157 bipolar outflow
}

\author{
J. Eislöffel ${ }^{1}$, B. Nisini ${ }^{2}$, R. Güsten ${ }^{3}$, H. Wiesemeyer ${ }^{3}$, and A. Gusdorf ${ }^{3,4}$ \\ 1 Thüringer Landessternwarte, Sternwarte 5, 07778 Tautenburg, Germany \\ e-mail: jochen@tls-tautenburg.de \\ 2 INAF-Osservatorio Astronomico di Roma, via di Frascati 33, 00040 Monteporzio Catone, Italy \\ 3 Max-Planck Institut für Radioastronomie, Auf dem Hügel 69, 53121 Bonn, Germany \\ ${ }^{4}$ LERMA, UMR 8112 du CNRS, Observatoire de Paris, École Normale Supérieure, 24 rue Lhomond, 75231 Paris Cedex 05, France
}

Received 27 January 2012 / Accepted 5 March 2012

\section{ABSTRACT}

\begin{abstract}
Context. Carbon monoxide is an excellent tracer of the physical conditions of gas in molecular outflows from young stars. Aims. To understand the outflow mechanism we need to investigate the origin of the molecular emission and the structure and interaction of the outflowing molecular gas. Deriving the physical parameters of the gas will help us to trace and understand the various gas components in the flow.

Methods. We observed CO (12-11) line emission at various positions along the L1157 bipolar outflow with GREAT aboard SOFIA. Results. Comparing these new data with CO (2-1), we find basically constant line ratios along the outflow and even at the position of the source. These line ratios lead us to estimates of $10^{5}$ to $10^{6} \mathrm{~cm}^{-3}$ for the gas density and 60 to $100 \mathrm{~K}$ for the gas temperature of the outflowing gas.

Conclusions. The constrained density and temperature values indicate that we are mostly tracing a low-velocity gas component everywhere along the outflow, which is intermediate between the already known cold gas component, which gets entrained into the flow, and the hot gas, which gets shocked in the outflow.
\end{abstract}

Key words. ISM: jets and outflows - stars: formation - stars: pre-main sequence - stars: low-mass - infrared: ISM - stars: mass-loss

\section{Introduction}

Carbon monoxide is widely considered to be the best probe of excitation conditions in the interstellar and protostellar molecular gas because of its high abundance and simple chemistry. In the study of outflows from young protostars, in particular, the $\mathrm{CO}$ molecule plays a central role. Low- $J$ rotational $\mathrm{CO}$ lines are routinely used to study the kinematics and energetics of the molecular gas swept-out by the protostellar jets and winds. At the same time, high- $J$ rotational $\mathrm{CO}$ lines, observable in the farIR wavelength range, are ideal tracers of the gas that is currently being shocked in the interaction of the jets with the ambient medium, which significantly contributes to the overall shocked gas cooling (e.g. Giannini et al. 2001).

In this contribution we report the first observation of a higher- $J$ line of $C O$, namely the CO (12-11) line, along various shocked spots in the L1157 bipolar outflow that were obtained with the GREAT heterodyne spectrometer ${ }^{1}$ (Heyminck et al. 2012) aboard SOFIA (Stratospheric Observatory For Infrared Astronomy), during three flights in its Early Science phase (Young et al. 2012).

The CO (12-11) transition lies at $365 \mathrm{~K}$ above the ground state and it is therefore a very good tracer of warm molecular gas. As such, it can be used to bridge the gap between the low-lying

\footnotetext{
${ }^{1}$ GREAT is a development by the MPI für Radioastronomie and the KOSMA/Universität zu Köln, in cooperation with the MPI für Sonnensystemforschung and the DLR Institut für Planetenforschung.
}

CO lines, which are related to the swept-out outflow material, and the higher- $J$ lines excited in the shocked hot gas.

The L1157 outflow is an ideal target for this pilot study with SOFIA because it is one of the most active and extensively studied outflows from low-mass young protostars. Observations in a wide range of wavelengths indicate the presence of strong chemical and physical gradients within the flow. Chemically, the outflow is extremely rich, with more than 20 molecular species detected, showing significant morphological variations along the flow (e.g. Bachiller et al. 2001). This chemical differentiation is associated with the presence of shock spots that show a range of excitation conditions that are probed, e.g., by multiwavelength $\mathrm{H}_{2}$ observations (Davis \& Eislöffel 1995; Caratti o Garatti et al. 2006; Nisini et al. 2010a). These shocked regions show up prominently in $\mathrm{H}_{2} \mathrm{O}$ emission as well, indicating that a warm gas-phase chemistry is actively taking place (Nisini et al. 2010b).

The outflow has been fully mapped in $\mathrm{CO}(2-1)$ by Bachiller et al. (2001), while sub-mm CO observations have so far only been obtained in the blueshifted southern lobe. In particular, Hirano \& Taniguchi (2001) have obtained spectra of CO (65), $\mathrm{CO}$ (4-3), and $\mathrm{CO}$ (3-2) in three different shock positions, suggesting that the bulk of the emission comes from gas at $n \sim$ $10^{4} \mathrm{~cm}^{-3}$ and $T \sim 50-150 \mathrm{~K}$. Herschel observations obtained by Codella et al. (2010) and Lefloch et al. (2010) suggested that the CO (5-4) emission in the brightest blueshifted shock spot (called B1 after Bachiller et al. 2001) can be divided into different velocity components with different excitation conditions: the 


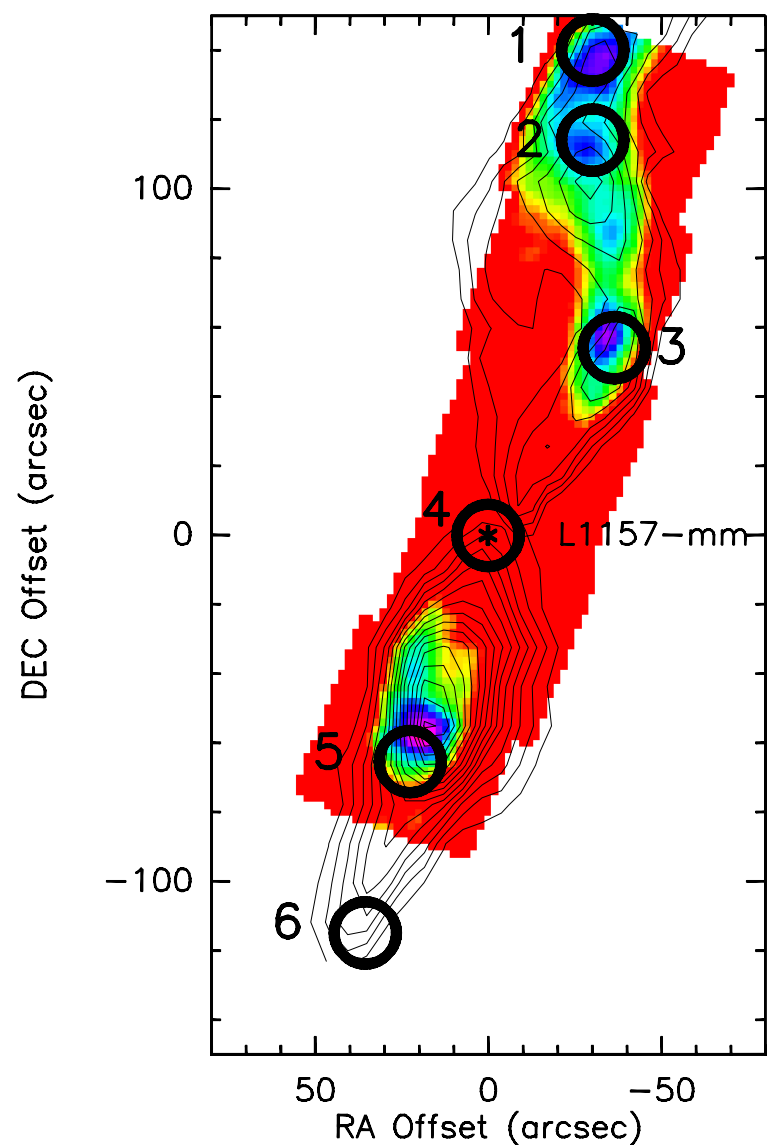

Fig. 1. Positions observed with SOFIA in the L1157 bipolar outflow, which is emanating from the embedded Class 0 source L1157-mm. The background colour map shows an image in the $\mathrm{H}_{2} 0-0 \mathrm{~S}(1)$ line at $17 \mu \mathrm{m}$ (from Nisini et al. 2010b) with superposed contours of the integrated CO (2-1) emission (from Bachiller et al. 2001). The targeted positions of the presented CO (12-11) observations with GREAT are labelled and indicated with circles, with diameters equal to the actual beam size of $21 . .3$ of the GREAT beam.

low-velocity gas at $n \sim 3 \times 10^{5} \mathrm{~cm}^{-3}, T \sim 100 \mathrm{~K}$, and the highvelocity gas at $n \sim 10^{4} \mathrm{~cm}^{-3}$ and $T>400 \mathrm{~K}$.

CO transitions at higher $J$ (from $J=15$ up to $J=20$ ), which probe the hottest gas component, have been detected by ISO at low spatial resolution and suggested the presence of CO gas with temperatures in the range 300-1000 K (Giannini et al. 2001). Very recently, high spatial resolution, non-velocity resolved PACS observations of CO lines from $J=14$ to 22 have been reported for the L1157-B1 position, which allows one to localize the hot gas in a compact shock of about $9^{\prime \prime}$ in size and to better constrain its physical properties (Benedettini et al. 2012).

\section{Observations and data reduction}

Data of the CO (12-11) line were obtained with GREAT at various positions in L1157. During SOFIA flights starting out of Palmdale, CA, on 14 July 2011 positions on the central source and towards the southern outflow lobe were obtained, while on the 28 and 30 September 2011 positions towards the northern outflow lobe were observed. Figure 1 shows the six targetted positions on a $\mathrm{H}_{2} 0-0 \mathrm{~S}(1)$ and $\mathrm{CO}(2-1)$ map. They were selected because they are peaks in the $\mathrm{SiO}$ map of Bachiller et al. (2001). GREAT was in its L1a/L2 configuration in upper side band. The fast-Fourier transform spectrometers were used as backends for
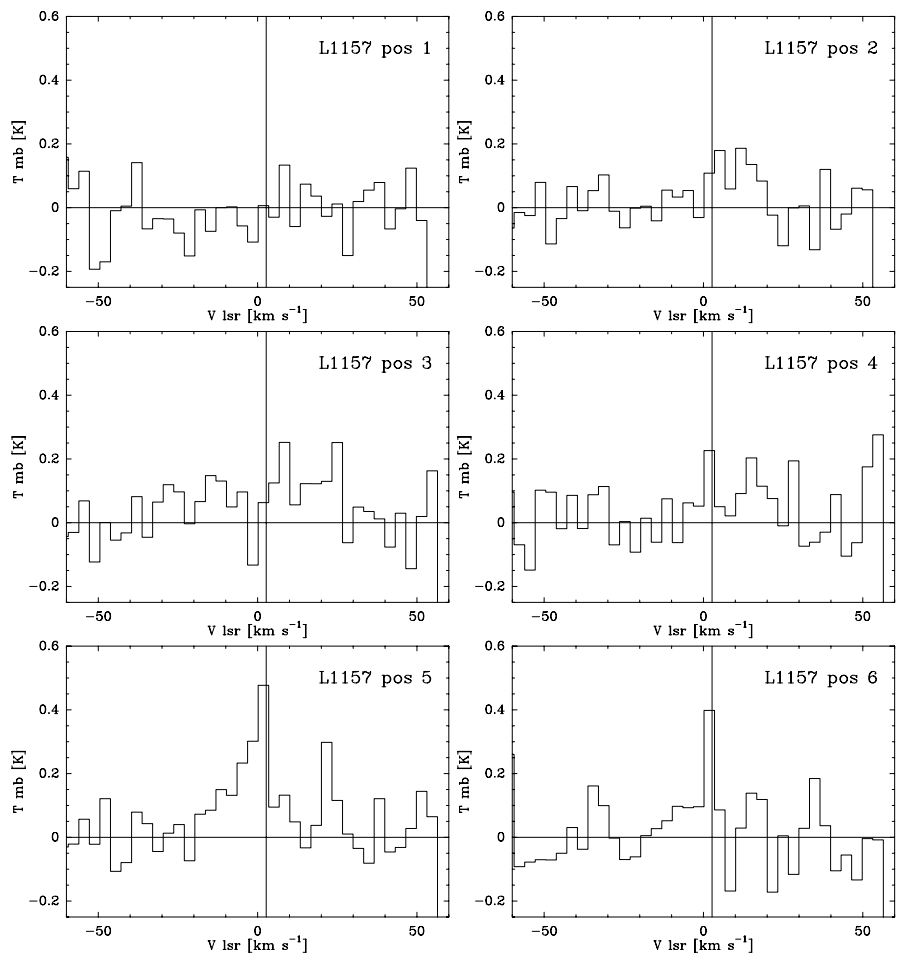

Fig. 2. Measurements of the $\mathrm{CO}(12-11)$ line in the six positions in
L1157 marked in Fig. 1. Data have been rebinned to velocity bins of $3.3 \mathrm{~km} \mathrm{~s}^{-1}$ for these diagrams. The vertical line marks the systemic velcocity of $v_{\mathrm{lsr}}=2.7 \mathrm{~km} \mathrm{~s}^{-1}$.

data recording, and data from the XFFTS backend are shown here.

The chop amplitude was $80^{\prime \prime}$ to the east and west of the source position. The observations were calibrated with the chopper wheel method using cold and warm loads (Heyminck et al. 2012). Bandpass-averaged zenithal opacities varied from $\tau=$ 0.11 to 0.30 , with averages of $0.21,0.14$, and 0.17 on the flights of 14 July, and 28 and 30, September, respectively.

The CLASS package ${ }^{2}$ was used for the standard data reduction, which included flagging of bad channels and atmospheric features, and a third order baseline subtraction. Data in the single positions were then averaged using a sigma weighting scheme, and calibrated for a forward efficiency of 0.95 and a main beam efficiency of 0.54 .

\section{Results}

The CO (12-11) line was observed towards the source position L1157-mm and five positions in the low-mass L1157 outflow (Fig. 1). The measured line fluxes at the various positions, integrated from $v_{\mathrm{lsr}}=-20 \mathrm{~km} \mathrm{~s}^{-1}$ to $+28 \mathrm{~km} \mathrm{~s}^{-1}$ as in Bachiller et al. (2001), are given in Table 1. CO (12-11) was detected in the inner four positions at a signal-to-noise ratio between 3.7 and 9.5. It was not detected in the northern-most position 1 and only marginally, at a signal-to-noise ratio of 2.3 , at the southernmost position 6 (Fig. 2). Although our data do not have at high signal-to-noise ratio, we find that at positions 2 and 3 our resolved line profiles are resembling those of Bachiller et al. (2001) in CO (2-1), with a peak near the systemic velocity at about $5 \mathrm{~km} \mathrm{~s}^{-1}$. A second peak around $10-20 \mathrm{~km} \mathrm{~s}^{-1}$, broader but weaker in the Bachiller et al. data, is seen in our data in these

2 see http://www.iram.fr/IRAMFR/GILDAS 
Table 1. Measured positions and fluxes.

\begin{tabular}{|c|c|c|c|c|c|c|c|c|}
\hline \multirow[t]{2}{*}{ Pos } & \multicolumn{2}{|c|}{ Position offset $^{a}$} & \multirow{2}{*}{$\begin{array}{l}T_{\text {int }}^{b} \\
{[\mathrm{~min}]}\end{array}$} & \multirow{2}{*}{$\begin{array}{c}\mathrm{rms} T_{\mathrm{mb}}{ }^{c} \\
{[\mathrm{~K}]}\end{array}$} & \multicolumn{2}{|c|}{$\int T \mathrm{~d} v \quad\left[\mathrm{~K} \mathrm{~km} \mathrm{~s}^{-1}\right]$} & \multirow{2}{*}{$\begin{array}{c}\text { Flux ratio } \\
\mathrm{CO}(12-11) / \mathrm{CO}(2-1)\end{array}$} & \multirow[t]{2}{*}{ Other designations } \\
\hline & $\Delta x\left[{ }^{\prime \prime}\right]$ & $\Delta y\left[{ }^{\prime \prime}\right]$ & & & $\operatorname{CO}(12-11)^{d}$ & $\mathrm{CO}(2-1)^{e}$ & & \\
\hline L1157-1 & -30.2 & 140.2 & 10.0 & 0.12 & $<1.9^{f}$ & 64.5 & $<0.04$ & L1157-R \\
\hline L1157-2 & -30.2 & 114.2 & 16.0 & 0.05 & $2.5(0.4)$ & 70.1 & $0.03 \quad(0.005)$ & L1157-R1 \\
\hline L1157-3 & -36.5 & 54.2 & 9.3 & 0.10 & $4.5(0.9)$ & 75.5 & $0.05 \quad(0.01)$ & L1157-R0 \\
\hline L1157-4 & 0.0 & 0.0 & 10.0 & 0.11 & $3.2(0.9)$ & 42.2 & $0.07 \quad(0.02)$ & L1157-mm \\
\hline L1157-5 & 22.8 & -65.3 & 8.1 & 0.09 & $6.7(0.7)$ & 95.1 & $0.06 \quad(0.006)$ & L1157-B1 \\
\hline L1157-6 & 35.4 & -114.8 & 7.4 & 0.16 & $2.9(1.3)$ & 41.0 & $0.07 \quad(0.03)$ & L1157-B2 \\
\hline
\end{tabular}

Notes. ${ }^{(a)}$ Position offsets are relative to the central source L1157-mm at RA $20^{\mathrm{h}} 39^{\mathrm{m}} 06.2 \mathrm{Dec}+68^{\circ} 02^{\prime} 15^{\prime \prime} .8(\mathrm{~J} 2000)$; ${ }^{(b)}$ total on-source integration time; ${ }^{(c)}$ measured in the range from $-90 \mathrm{~km} \mathrm{~s}^{-1}$ to $-50 \mathrm{~km} \mathrm{~s}^{-1}$ because of an atmospheric feature on the red side beyond $+50 \mathrm{~km} \mathrm{~s}{ }^{-1}$; ${ }^{(d)}$ this paper; (e) from Bachiller et al. (2001), integrated from $-20 \mathrm{~km} \mathrm{~s}^{-1}$ to $+28 \mathrm{~km} \mathrm{~s}^{-1}$; (f) $2-\sigma$ upper limit.

positions as well. A similar, but narrower peak is also present in position 5, where a secondary component is not seen in the low- $J$ CO spectra (e.g. Lefloch et al. 2010). We obtained the clearest detection towards position 5 . In this position, which corresponds to the bright shocked spot L1157-B1, the emission shows a peak close to the systemic velocity of $2.7 \mathrm{~km} \mathrm{~s}^{-1}$, and exhibits a blue wing that we can trace to about $-20 \mathrm{~km} \mathrm{~s}^{-1}$.

In their analysis of low- $J$ rotational CO emission in L1157B1, Lefloch et al. (2010) detected two velocity components in the lines: a fainter high velocity component (HVC) blueward of $v_{\text {lsr }}=-7.5 \mathrm{~km} \mathrm{~s}^{-1}$, and a brighter low velocity component (LVC) redward of $v_{\mathrm{lsr}}=-7.5 \mathrm{~km} \mathrm{~s}^{-1}$. Our new observations in CO (1211) are therefore mainly tracing the LVC.

\section{Discussion}

It is interesting to use the new data to estimate the physical conditions of the emitting gas along the L1157 outflow, to infer from them the origin of the emission, and to see if these conditions are changing along the flow.

To infer the physical conditions giving rise to the CO (1211) emission observed with GREAT, we compared it with the observations of Bachiller et al. (2001), who mapped the entire L1157 outflow in CO (2-1). Their CO (2-1) map was obtained at the IRAM 30-m antenna with a beamsize of $11^{\prime \prime}$. For the purpose of our comparison we convolved this map to a beamsize of $21 . \prime 3$ - corresponding to that of GREAT for the CO (12-11) line - and measured the emission in the positions also observed with GREAT, integrated over a velocity range from $-20 \mathrm{~km} \mathrm{~s}^{-1}$ to $+28 \mathrm{~km} \mathrm{~s}^{-1}$, as in Bachiller et al. (2001). These values are reported in Table 1, Col.7. We integrated the emission of the CO (12-11) line over the same velocity range (Table 1, Col. 6, with errors in brackets). The corresponding line ratio of $\mathrm{CO}$ (12$11) / \mathrm{CO}(2-1)$ is also reported in Table 1. Its associated error is dominated by the rms noise of our CO (12-11) observations.

The first view shows that the CO (12-11)/CO (2-1) line ratio is fairly constant from the source to the innermost northern knot L1157-R0 and along the knots in the southern outflow lobe, with an average value of about $0.06 \pm 0.01$. The ratio may be decreasing for the two northern-most knots, but this difference is not very significant, given the associated errors, and because for the northern knot L1157-R we can only give a 3- $\sigma$ upper limit, because of the non-detection of CO (12-11).

It is interesting to note that the $\mathrm{CO}$ line ratio of 0.07 observed for L1157-mm, that is "on-source", is not significantly different from that of the outflow positions. This suggests that the onsource $\mathrm{CO}$ emission is also dominated by shock emission, and not by emission from the envelope of the embedded source. We will investigate this in more detail below.

To derive information about the physical conditions of the $\mathrm{CO}$ emitting gas in the observed positions along the L1157 outflow, we used the radiative transfer model RADEX (van der Tak et al. 2007) to compute the expected CO (12-11)/CO (2-1) ratio to confront it with our observations. We adopted a planeparallel large velocity gradient (LVG) approximation and the collisional coefficients of Yang et al. (2010), and - given the similar flux ratios everywhere - are assuming that the emission regions of both lines must be very similar.

With a single line ratio at hand, it is obvious that the range of free parameters has to be restricted with additional information to break the otherwise existing strong degeneracies between gas density and temperature. To this end, we are considering a column density of the CO gas that is compatible with the Spitzer observations of pure rotational lines of molecular hydrogen of the same regions as we observed here. Nisini et al. (2010b) estimated that the total column density of $\mathrm{H}_{2}$ associated with the shocked spots is of the order of $10^{20} \mathrm{~cm}^{-2}$, with variations of only a factor of a few in the various regions. Assuming a $\mathrm{CO}$ abundance of $10^{-4}$, this translates into a column density of about $10^{16} \mathrm{~cm}^{-2}$ for the CO gas. We also assumed a value for the velocity dispersion $\Delta v$ of about $10 \mathrm{~km} \mathrm{~s}^{-1}$. This is what we see in our spectrum at position 5 as the width of the line thanks to the high spectral resolution of GREAT.

In Fig. 3, we show (in black) the predicted CO (12$11) / \mathrm{CO}(2-1)$ line ratio as a function of the kinetic temperature and for total $\mathrm{H}_{2}$ densities of $10^{4}, 10^{5}$, and $10^{6} \mathrm{~cm}^{-3}$. Because the line ratios derived from our $\mathrm{CO}(12-11)$ observations and those from the map of Bachiller et al. (2001), that are listed in Table 1 are so similar for the various positions along the flow, they are shown as a black stripe here for clarity.

Assuming an $\mathrm{H}_{2}$ density of $10^{4}$ to $10^{6} \mathrm{~cm}^{-3}$, we can constrain the kinetic temperature of the observed $\mathrm{CO}$ gas to about 60 to $200 \mathrm{~K}$. In their analysis of the single, brightest position L1157-B1, Lefloch et al. (2010) estimated a gas density of $n\left(\mathrm{H}_{2}\right)$ $\sim 3 \times 10^{5} \mathrm{~cm}^{-3}$ and a temperature of $T \sim 100 \mathrm{~K}$ for the gas in the low-velocity component. Comparing the estimate from our newly observed line ratio with theirs, we find both results to be consistent. This confirms that we are mostly tracing the LVC component along the whole flow, that is intermediate between the cold gas, which gets entrained into the flow, and the hot, shocked gas.

We already mentioned the surprising fact that the CO (1211)/CO (2-1) line ratio at the position of the central source L1157-mm is similar to the ratios we are finding along the flow. An additional constraint for the $\mathrm{CO}$ gas at this central position 


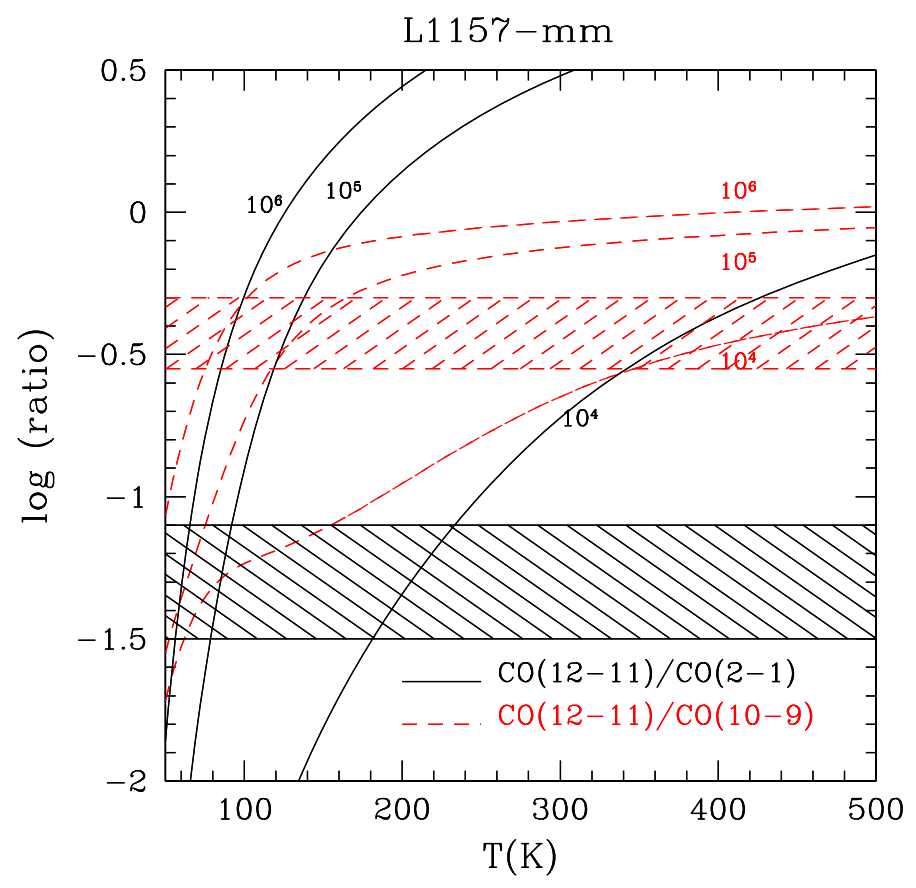

Fig. 3. Ratios of the integrated fluxes in CO (12-11)/CO (2-1) versus gas temperature for a $\mathrm{CO}$ gas column density of $10^{16} \mathrm{~cm}^{-2}$ at the various observed positions in the L1157 outflow (in black). For the observed position of the central source L1157-mm the ratios for CO (12$11) / \mathrm{CO}(10-9)$ are given as well (in red).

can be provided by an observation of the CO (10-9) line obtained with the HIFI spectrometer aboard Herschel (Yildiz et al., in prep.). This observation was obtained with a beamsize of $20^{\prime \prime}$, which is comparable to the beamsize of GREAT for the CO (1211) line. It gives a flux integrated over the same velocity range as our observations of $8.4 \pm 0.4 \mathrm{~K} \mathrm{~km} \mathrm{~s}^{-1}$. This then implies a CO (12-11)/CO (10-9) line ratio of $0.4 \pm 0.1$. In Fig. 3 , we show the model calculations with RADEX for the line ratio of CO (12-11)/CO (10-9) (in red) together with that of CO (1211)/CO (2-1) (in black). The assumed column density is again $10^{16} \mathrm{~cm}^{-2}$, and gas densities of $10^{4}, 10^{5}$, and $10^{6} \mathrm{~cm}^{-3}$ are plotted as lines. The observed line ratios and their associated errors are shown as black solid-lined and red dashed bands.

Figure 3 shows that a low-density solution would not be able to simultaneously account for the two line ratios: the CO (12$11) / \mathrm{CO}(2-1)$ ratio would imply a $\mathrm{CO}$ gas temperature of 200 to $240 \mathrm{~K}$, while the $\mathrm{CO}(12-11) / \mathrm{CO}(10-9)$ would require gas in excess of $350 \mathrm{~K}$. Assuming that both line ratios are tracing the same bulk mass of gas, densities of between $10^{5}$ and $10^{6} \mathrm{~cm}^{-3}$ and gas temperatures of about 60 to $100 \mathrm{~K}$ are required.

This analysis with the additional use of the $\mathrm{CO}$ (1211)/CO (10-9) line ratio thus confirms and strenghtens the gas parameters that we found from the single line ratio along most of the L1157 outflow, and the estimates of Lefloch et al. (2010) for position L1157-B1 in the flow. It supports our earlier finding that the $\mathrm{CO}$ emission in the on-source position is also dominated by shocked gas, similar to what we see along the outflow, and not by cold emission from an envelope.

Hirano \& Taniguchi (2001) analysed spectra of the $\mathrm{CO}$ (6-5), CO (4-3), and CO (3-2) lines in three different shock positions in L1157. They found that the emission mostly comes from gas at about $n \sim 10^{4} \mathrm{~cm}^{-3}$ and $T \sim 50-150 \mathrm{~K}$. From our SOFIA observations we derive a similar temperature range. For the central position, however, a higher density of the order of $10^{5}$ to $10^{6} \mathrm{~cm}^{-3}$ is indicated.

Benedettini et al. (2012) have analysed the velocityintegrated $\mathrm{CO}$ emission from $J=14-13$ to $J=22-21$ observed in position B1 (our position 5) with Herschel. They derived that these lines are compatible with gas at $200<T<800 \mathrm{~K}$ and $n \geq 10^{5} \mathrm{~cm}^{-3}$. Clearly, CO emission at higher $\mathrm{J}$ is dominated by a gas warmer than what we are inferring here, testifying that the emission of the series of lines of the $\mathrm{CO}$ ladder is able to trace the temperature stratification that is present across the shocked gas in the outflow.

\section{Conclusions}

We presented observations of the $\mathrm{CO}(12-11)$ line at various positions along the low-mass L1157 bipolar outflow obtained with GREAT aboard SOFIA.

Comparing these new $\mathrm{CO}(12-11)$ data with $\mathrm{CO}(2-1)$, we found that the line ratios are nearly constant along the flow, even at the position of the outflow source. An estimate of the physical parameters of the emitting gas shows that we are most likely tracing matter with a total gas density of about $10^{5}$ to $10^{6} \mathrm{~cm}^{-3}$ and at a temperature between 60 to $100 \mathrm{~K}$.

This indicates that we are mostly tracing a low-velocity gas component in the outflow, which is intermediate between the cold gas that gets entrained into the outflow and is seen in low- $J$ rotational $\mathrm{CO}$ lines, and the hot gas in the shocks in the outflow that was seen in very high- $J$ rotational CO lines with ISO.

Acknowledgements. We thank Rafael Bachiller for making his CO (2-1) map of L1157 available to us. We thank Umut Yildiz for making the information on the $\mathrm{CO}(10-9)$ integrated emission available to us prior to publication. Based [in part] on observations made with the NASA/DLR Stratospheric Observatory for Infrared Astronomy. SOFIA Science Mission Operations are conducted jointly by the Universities Space Research Association, Inc., under NASA contract NAS2-97001, and the Deutsches SOFIA Institut und DLR contract 50 OK 0901. $\mathrm{JE}$ is grateful for a travel grant from the German Aerospace Center DLR, which allowed him to be onboard SOFIA for the first flight, on which data for this programme were obtained.

\section{References}

Bachiller, R., Pérez Gutiérrez, M., Kumar, M. S. N., \& Tafalla, M. 2001, A\&A, 372,899

Benedettini, M., Busquet G., Lefloch B., et al. 2012, A\&A, 539, L3

Caratti o Garatti, A., Giannini, T., Nisini, B., \& Lorenzetti, D. 2006, A\&A, 449, 1077

Codella, C., Lefloch, B., Ceccarelli, C., Cernicharo, J., Caux, E., Lorenzani, A., Viti, S. et al. 2010, A\&A, 518, L112

Davis, C. J., Eislöffel, J. 1995, A\&A, 300, 851

Giannini, T., Nisini, B., \& Lorenzetti, D. 2001, ApJ, 555, 40

Heyminck, S., Graf, U. U., Güsten, R., et al. 2012, A\&A, 542, L1

Hirano, N., \& Taniguchi, Y. 2001, ApJ, 550, L219

Lefloch, B., Cabrit, S., Codella, C., et al. 2010, A\&A, 518, L113

Nisini, B., Benedettini, M., Codella, C., et al. 2010a, A\&A, 518, L120

Nisini, B., Giannini, T., Neufeld, D. A., et al. 2010b, ApJ, 724, 69

van der Tak, F. F. S., Black, J. H., Schöier, F. L., Jansen, D. J., \& van Dishoeck,

E. F. 2007, A\&A, 468, 627

Yang, B., Stancil, P. C., Balakrishnan, N., Forrey, R. C. 2010, ApJ, 718, 106

Young, E. T., Becklin, E. E., Marcum, P. M., et al. 2012, ApJ, 749, L17 